\title{
Rare Diagnosis of Benign Schwannomas after Right Thyroid Lobectomy in A 12-Year-Old Female
}

\author{
Samantha Alsop, Bennett Berning, Carolina Paredes-Molina, Chloe French, Tyler Mouw, Kurt Schropp \\ and Peter DiPasco* \\ University of Kansas Medical Center, Department of Surgery, United States
}

Received: November 11, 2017; Published: November 28, 2017

*Corresponding author: Peter DiPasco, University of Kansas Medical Center, Department of Surgery, Mailstop 2005, 3901 Rainbow Blvd., Kansas City, KS 66205, United States, Tel: 913-217-8706; Email: pdipasco@kumc.edu

\begin{abstract}
Schwannomas are benign neoplasms arising from neural sheath cells. Schwannomas most commonly arise in the head and neck region, most commonly developing from the vestibulocochlear nerve. On rare occasions, primary schwannomas have been described originating from the thyroid. Traditional diagnostic modalities are often ineffective in diagnosing schwannomas of the thyroid preoperatively, leading to an incidental finding after surgical intervention has occurred. The case we present is of a 12-year-old female who underwent a right thyroid lobectomy after two fine needle aspirations that raised concern for follicular malignancy, Hurthle cell type. Final pathology revealed a benign, S-100 positive, primary schwannoma of the thyroid.
\end{abstract}

Keyword: Thyroid Schwannoma

\section{Introduction}

Schwannomas are benign, peripheral nerve neoplasms originating from neural sheath cells (Schwann cells) [1]. These well-circumscribed, encapsulated masses develop from nerve roots throughout the body, with $25 \%$ to $45 \%$ originating in the head and neck [2]. In a majority of cases, benign Schwannomas arise from the vestibulocochlear nerve (CN VIII), followed by trigeminal nerve (CN V) and facial nerve (CN VII) [2]. On rare occasions primary Schwannomas have been reported in the thyroid gland, with the first case reported in the literature in 1964 [3]. Since then, a vast majority of cases reported of schwannomas involving the thyroids are in adult patients. In 2004, one case was published involving a twelve-year-old female [4].In 2010, a primary schwannoma of the thyroid was reported in a fourteen-year-old male [5]. The case we present is an additional schwannoma of the thyroid in a twelveyear-old female.

\section{Case Report}

A twelve-year-old female patient first presented to the University of Kansas Pediatric Department with increased rightsided neck swelling after experiencing recent upper respiratory tract infection. The neck was non-tender. There was no reported dysphagia, dysphonia or hoarseness and no hyperthyroid or hypothyroid symptoms were noted. Past medical history was only significant for mild asthma with occasional use of an albuterol inhaler. No surgical history was reported and no family history of thyroid disorders or thyroid cancer. The patient did not smoke or consume alcohol. Physical exam revealed right neck swelling that was non-tender to palpation. Thyroid function tests were within normal limits. An ultrasound was ordered and revealed a right thyroid hypoechoic mass in the middle portion of the thyroid at and along the isthmus measuring $2.3 \mathrm{~cm}$ by $1.4 \mathrm{~cm}$ by $1.8 \mathrm{~cm}$ (Figure 1). The margins were slightly lobulated and irregular with some vascularity seen at the inferior medial edge. Mild mass effect was noted on the adjacent trachea with no evidence of tracheal invasion. The left thyroid lobe was unremarkable. Small, normal appearing lymph nodes were noted within the neck. At this point, the differential diagnosis included a complex hemorrhagic cyst versus a hypovascular solid thyroid neoplasm.

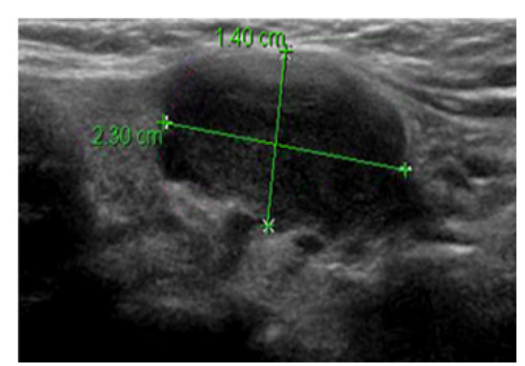

Figure 1: A right thyroid hypoechoic mass in the middle portion of the thyroid at and along the isthmus measuring $2.3 \mathrm{~cm}$ by $1.4 \mathrm{~cm}$ by $1.8 \mathrm{~cm}$. 
The patient was referred to University of Kansas Pediatric Surgery for further evaluation. The patient continued to be asymptomatic with physical exam noting fullness of the right thyroid that was non-tender to palpation. At this time it was recommended that a biopsy by fine needle aspiration be obtained and a repeat ultrasound be completed in two months. A repeat thyroid ultrasound was obtained two months after the initial study and revealed a stable right thyroid mass in the medial right lobe and isthmus measuring $2.1 \times 1.5 \times 1.9$ (Figure 2). The differential continued to be hemorrhagic cyst versus benign or malignant neoplasm. A biopsy by fine needle aspiration was again encouraged. Three months following initial presentation a biopsy was obtained by fine needle aspiration. Cytology was suspicious for a follicular neoplasm, Hürthle cell type and the patient was referred to Surgical Oncology at the University of Kansas. During the evaluation by surgical oncology, the patient continued to report no dysphagia, dysphonia or hoarseness and no hyperthyroid or hypothyroid symptoms. Physical exam remained unchanged with a palpable, non-tender right-sided neck mass noted with no lymphadenopathy noted. Repeated thyroid function tests were again within normal limits. Because the patient presented at such a young age, the case was presented at a multidisciplinary endocrine tumor conference at the University of Kansas. After detailed discussion, it was recommended that a second biopsy by fine needle aspiration be obtained with Afirma molecular genetic testing.

The repeat fine needle aspiration was again suspicious for a follicular neoplasm, Hürthle cell type. The repeat fine needle aspiration using Afirma thyroid FNA analysis was suspicious for malignancy, with the probability of malignancy from $10-30 \%$ to $40 \%$. However, the test is not validated on patients under twentyone years old. The gene expression classifier also identified a genetic signature similar to malignant melanoma. As a result of the second fine needle aspiration, combined with the molecular genetic testing, surgical lobectomy with the possibility of bilateral neck exploration and total thyroid ectomy was recommended. The patient underwent right thyroid lobectomy with intraoperative monitoring of the recurrent laryngeal nerve. Surgical findings revealed an enlarged, firm, right inferior thyroid lobe with effacement of the anterolateral tracheal surface, requiring a shave dissection of the nodule off the tracheal rings (Figure 3). No lymphadenopathy was noted within the neck. Final pathology revealed a benign schwannoma in the right thyroid lobe (Figure 4). The mass was less than 4 centimeters, lacked mitotic activity, necrosis or nuclear pleomorphism, all of which are factors that support a benign cellular process. The tumor was positive for S-100 and negative for TTF-1, which supported diagnosis of benign schwannoma.

The case was again discussed in multidisciplinary endocrine tumor conference to determine adequate follow-up. Because pathology showed positive margins at the anterior tracheal margin, risk of recurrence remained. It was decided that the patient would be followed with a thyroid ultrasound six months postop to monitor for recurrence. Subsequent serial ultrasound evaluations over the following three years have demonstrated no evidence of recurrence and the patient continues to do well.

\section{Radiology Images (Figure 1-4)}

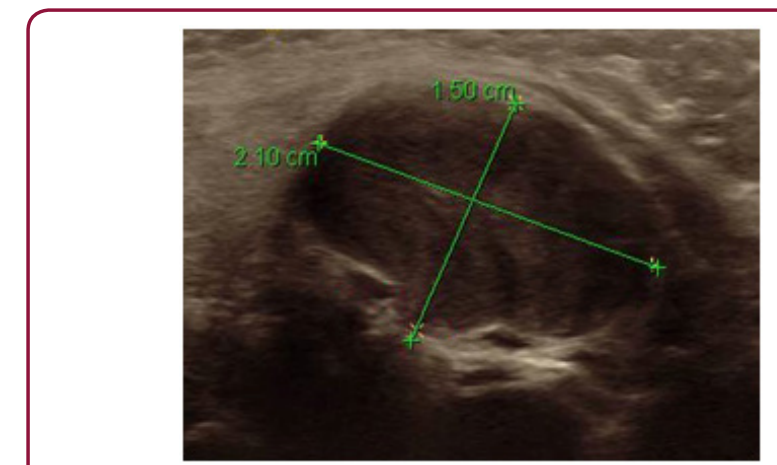

Figure 2: A stable right thyroid mass in the medial right lobe and isthmus measuring $2.1 \times 1.5 \times 1.9$.



Figure 3: Gross surgical specimen of the nodule off the tracheal rings.

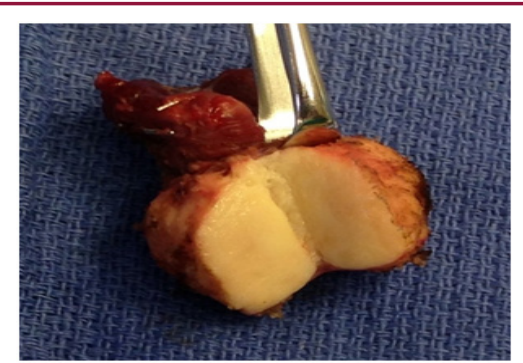

Figure 4: Gross surgical specimen of a benign schwannoma in the right thyroid lobe.

\section{Discussion}

Primary schwannomas of the thyroid gland are exceptionally rare. To our knowledge, less than twenty cases have been reported in the literature, with only two other cases reported involving a child. One case involving a fourteen-year old male [5] and our case being the second case reported of a primary schwannoma in a twelveyear-old female. Fine needle aspiration is the foundation of initial management of a thyroid nodule. The technique has reduced the number of thyroid ectomies and increased the number of malignant diagnoses after thyroidectomy [6]. In the case presented; fine needle aspiration was performed on two occasions. Each fine needle aspiration was suspicious for follicular neoplasm, Hürthle cell type. The results of the fine needle aspiration combined with the ultrasound images raised concern for malignancy, which lead to surgical intervention. It has been suggested that multiple diagnostic modalities combined with fine needle aspiration 
should be considered. Ultrasound, CT and MRI can be used to help differentiate between solid and cystic masses, but cannot be used to obtain a definitive diagnosis [7]. PET-CT has also been described as having a possible role in pre-operative diagnosis of thyroid schwannomas. It has been shown that both benign schwannomas and malignant peripheral nerve sheath tumors show FDG uptake [8]. This may be useful when distinguishing between schwannoma and other benign masses of the thyroid where FDG uptake would not occur [7], but would have been of little use in our case, due largely to the fact that malignancy was our greatest concern. Recurrence of schwannomas is uncommon and due largely to the inability to fully resect the tumor. Although rare, malignant transformation from benign schwannoma in sites outside the thyroid has been reported in the literature. For this reason we have decided to follow up with ultrasound every six months.

\section{Conclusion}

In conclusion, schwannomas originating from the thyroid gland are rare and difficult to diagnose pre-operatively. They frequently present as a painless neck mass and are difficult to distinguish in appearance from most thyroid nodules on ultrasound. Definitive diagnosis of a thyroid schwannoma prior to thyroid lobectomy is an uncommon occurrence and is often found incidentally by pathology after surgical intervention.

\section{References}

1. Brunicardi C, Andersen T, Dunn D, Hunter J, Pollock R (2006) Schwartz's Manual of Surgery, ( $8^{\text {th }}$ edn).

2. Uri O, Baron E, Lefel O, Bitterman A (2009) Primary schwannoma of the thyroid gland presenting as an asymptomatic cold nodule. Am J Otolaryngol 30(6): 427-429.

3. Jayaram G (1999) Neurilemmoma (schwannoma) of the thyroid diagnosed by fine needle aspiration cytology. ActaCytol 43(4): 743-744.

4. Baglaj M, Markowska-Woyciechowska A, Sawicz-Birkowska K, Dorobisz U (2004) Primary neurilemmoma of the thyroid gland in a 12-year-old girl. J PediatrSurg 39(9): 1418-1420.

5. An J, Oh YL, Shin JH, Jeong HS (2010). Primary schwannoma of the thyroid gland: a case report. ActaCytol 54(5 Suppl): 857-862.

6. Datta RV, Petrelli NJ, Ramzy J (2006) Evaluation and management of incidentally discovered thyroid nodules. SurgOncol 15(1): 33-42.

7. Knulst R, WM Bosman, Ewan D Ritchie, Andy da Costa (2014) Cystic schwannoma of the recurrent laryngeal nerve: a rare finding posing diagnostic difficulties. BMJ Case Rep 2014.

8. Beaulieu S, Rubin B, Djnag D, Conrad E, Turcotte E, et al. (2004) Positron emission tomography of schwannomas: emphasizing its potential in preoperative planning. AJR Am J Roentgenol 182(4): 971-974.

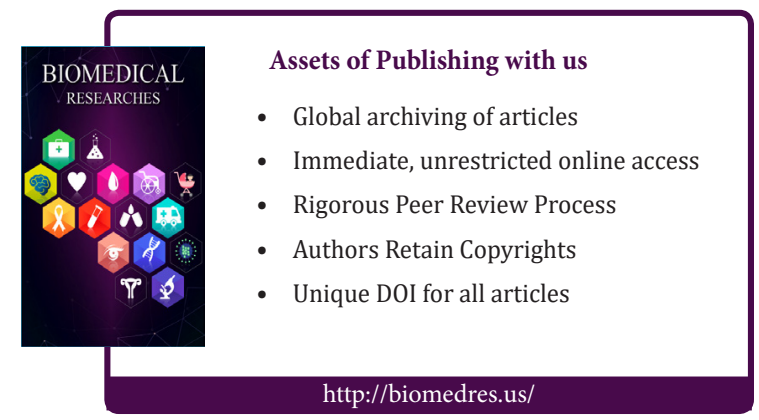

